\title{
Interobserver Variability in Mitotic Count for Meningioma Grading: How Can We Reduce It?
}

\author{
Ismail SAYGIN, Emel CAKIR, Mustafa Emre ERCIN \\ Karadeniz Technical University, Faculty of Medicine, Department of Pathology, Trabzon, Turkey
}

Corresponding author: Ismail SAYGIN ismailsaygin@ktu.edu.tr

\section{ABSTRACT}

AIM: To evaluate the interobserver variability in determining the number of mitoses in 10 high-power field (HPF) and thus the tumor grade, and to investigate how to reduce grade discordance between the observers and the most useful method to identify the patients who would receive an additional treatment.

MATERIAL and METHODS: Two hundred and seventy cases with meningioma were re-evaluated by three experienced pathologists and five senior residents. They determined the number of mitotic figures in 10 HPF in each slide. Re-evaluation of the cases, which were found of different grades from the reference observers was requested by full scan method. Statistical analysis was performed by using SPSS V23.0.

RESULTS: A moderate agreement was found between the observers and the reference observer. After the evaluation of mitotic activity with the full scan method, the mean numbers of mitoses found by the observers in 10 HPF were increased. In the first evaluation, 4-6 cases were defined as Grade II by the observers. Whereas, 23-27 cases were defined as Grade II after the full scan method.

CONCLUSION: If there are less than 16 mitotic figures throughout the slide, it is more difficult to find the 10 HPF including 4 or more mitosis. Interobserver variability in mitotic figure counting can be reduced by full scan method, and examining the hematoxylin and eosin stained slides by the full scan method helps us to determine the true histologic grade of meningiomas in most cases, who would receive an additional treatment.

KEYWORDS: Brain neoplasm, Grade concordance, Interobserver variability, Meningioma, Mitosis

\section{INTRODUCTION}

$\mathrm{M}$ eningioma is the most common primary brain tumor of the central nervous system (11). Fifteen different morphological types of meningiomas have been described so far. Although there are several morphological types, most of the meningiomas are relatively slow-growing and Grade I tumors, with a good prognosis. According to the World Health Organization (WHO) 2016 histological criteria, up to $15 \%$ of the meningiomas are atypical and $2 \%$ are anaplastic. The grading of meningiomas is primarily based on morphological parameters, mitotic count (mitosis in 10 consecutive high-power field (HPF) $<4=$ Grade 1, 4-9 = Grade 2 , and $\geq 20=$ Grade 3), and brain invasion. Mitotic index is the only quantitative criteria used in the grading of meningiomas.
Meningiomas have so far been classified based on histological and cytological features. The aim of grading meningiomas is to predict tumor recurrence or progression, as well as guide the therapeutic management, prolonging the survival of the patients. In meningioma, grade and complete surgical resection are still the best indicators of progression-free survival. Therefore, grading is of great importance, both to accurately predict the survival as well as to determine the accurate additional therapies.

Several factors are considered in the treatment of meningiomas. The most important ones are: tumor size, localization, patient age, general health status, and the therapeutic target. Not all patients with meningiomas need to be urgently treated. Small or slow-growing meningiomas that do not cause symptoms 
are monitored by computed tomography. If the tumor size increases or causes symptoms, treatment options are considered. In that case, surgery is the first choice for therapy. The purpose of the surgery is total resection of the tumor. If this is not possible or if the lesion is close to vital structures, it should be removed as much as possible.

If the tumor is totally excised surgically, it may not require an additional treatment. If total excision of the tumor is not possible and if pathological examination reveals that the tumor is low grade (Grade I), only periodic follow-up is recommended. In contrast, radiotherapy (RT) is required if the tumor relapses after surgery or exhibits atypical or malignant features on pathological examination. In some cases, an RT called stereotactic radiosurgery may be preferred for small residual tumors.

Grade I meningiomas can be cured by surgical resection depending on the tumor localization. However, some meningiomas show recurrence and malignant transformation even if they are totally removed. According to the Simpson Grading System, the amount of resection closely correlates with a progression-free survival (6).

Adjuvant RT has shown to increase the disease-free survival and overall survival in atypical and anaplastic meningiomas, especially after subtotal resection. Its effect in Grade I meningioma is, however, controversial. Chemotherapy is not preferred to treat meningiomas, but it can be used in cases that do not respond to surgery and radiation.

Some meningioma cases defined as Grade I can recur $(5,18)$. Furthermore, the results of some studies show that some patients may be misdiagnosed and receive insufficient treatment. In contrast, few studies have also proposed that an adjuvant RT is not required for all Grade II meningiomas $(15,16)$.

In histopathological grading of meningiomas, determining an accurate mitotic index is one of the most important factors. Increased mitotic count is associated with a higher grade and, thus, a worse prognosis. Ho et al. showed that $\geq 1.5$ / $\mathrm{mm}^{2}$ mitotic activity rate was one of the best indicators of recurrence (7).

Several studies have been carried out to facilitate the counting of mitotic activities on hematoxylin and eosin ( $\mathrm{H}$ and $\mathrm{E}$ ) stained sections, a classical method. Using the $\mathrm{PHH} 3$ antibody is one of the most important methods for this purpose. However, some cells such as apoptotic bodies also get stained besides mitotic figures by this method, resulting in inaccurate counting of the mitotic figures, and thus this method is not routinely used. Nevertheless, in some studies, $\mathrm{PHH} 3$ antibody was used as an immunohistochemical marker to determine mitosis. The studies of Duregon et al. and Ribalta et al. revealed that the number of cells stained with $\mathrm{PHH} 3$ could be used instead of the number of mitotic figures found on $\mathrm{H}$ and $\mathrm{E}$ stained slides $(4,14)$, thus being a reliable marker for mitotic count with a high interobserver reproducibility. However, they proposed that there should be a threshold value to describe the grade by $\mathrm{PHH} 3$ because the number of cells stained with $\mathrm{PHH} 3$ exceeds the number of mitosis found in $\mathrm{H}$ and $\mathrm{E}$ stained slides (4). Kim at al. used $\mathrm{PHH} 3$ for mitosis count, and they determined that six stained cells with $\mathrm{PHH} 3$ was the threshold value for Grade II (8). The results of the study of Colman et al. suggested $\mathrm{PHH} 3$ staining to be a useful method in other neoplasms such as infiltrating astrocytoma (2). In addition to manual counting of mitotic figures, some new additional procedures based on convolutional neural networks are being developed in breast cancer (17).

In our daily routine practice, suspicious cases between Grade I and Grade II make our job harder. In our experience, these suspected cases have mitotic figures that are at the limit of sufficiency to be defined as Grade II. The number of mitoses in $10 \mathrm{HPF}$ is the most common cause of interobserver variability in meningiomas. One mitosis may change the grade of meningioma. Therefore, accurate determination of mitosis is very important to minimize interobserver variability.

The aim of this study was to evaluate the interobserver variability in determining the number of mitoses in $10 \mathrm{HPF}$ and thus the tumor grade. In case of interobserver variability, the aim was to find the reasons for these differences and determine potential solutions to reduce the variability. Thus, the cases with Grade II and III meningiomas would not be overlooked, and patients who are to receive an additional treatment will be determined accurately.

\section{MATERIAL and METHODS}

In the study, 270 cases with meningioma were included. The hematoxylin and eosin ( $\mathrm{H}$ and $\mathrm{E})$ stained slides of the cases were re-evaluated. Cases with brain invasion and with atypical morphological patterns such as clear cell, rhabdoid, papillary, and chordoid were excluded. Appropriate formalin-fixed paraffin-embedded blocks were selected. From the selected paraffin blocks, 5-micron thick sections were prepared and stained by an automated slide staining machine.

Cases were examined by reference observers ( 3 experienced pathologists who had been working in neuropathology for at least 4 years), firstly. For every case, all slides were examined. Then, the slide which was most representative of the mitotic count and grade was determined by the three experienced pathologists together. The representative slide was the most mitotically active one among all the slides. For examination, $\mathrm{H}$ and $E$ stained representative slides were divided into random groups, which included five slides each. The representative slides were given to the five senior residents (observers) for examination. Five slides were examined by each observer during his daily routine work. They determined the number of the mitotic figures in each slide. For this reason, the observers used a counting method, described in the textbook "2016 WHO Classification of Tumors of the Central Nervous System" and was used by Perry et al. too $(9,12)$. This method was based on the number of mitotic figures that were obtained in 10 consecutive HPFs. In the second part of the study, by a new method called "full scan," re-evaluation of the cases, which were found of different grades from the reference observers was requested. Full scan was the method based on screening of the whole slide at $400 \times$ magnification, finding all the mitotic 
figures in the slide, and then counting the mitotic figures in 10 consecutive HPFs, where they were located densely. Results were interpreted statistically.

\section{Statistical Methods}

IBM SPSS Statistics for Windows, Version 23.0 (IBM Corp. Released 2015; Armonk, NY: IBM Corp) was used for the analysis of the data. The descriptive statistics of the evaluation results were expressed as numbers and percentages for ordinal data, and as mean, standard deviation, minimum, and maximum for the interval data. The variables were investigated using One-Sample Kolmogorov-Smirnov test to determine normalcy of distribution. Correlation between dependent variables was analyzed by the Spearman correlation test and presented with scatter plot graphs. The interobserver agreement between the pathologists was investigated using the Kappa test. Kappa scores were interpreted as follows: values $\leq 0$ indicative of no agreement, $0.01-0.20$ indicative of none to slight, $0.21-0.40$ as fair, $0.41-0.60$ as moderate, $0.61-0.80$ as substantial, and $0.81-1.00$ as almost a perfect agreement. Online Kappa Calculator was used to determine the agreement between more than two variables (13). In the second part of the study, the interval data of two dependent variables were compared with the Wilcoxon test. The ordinal data of two dependent variables were compared with the McNemar test. The level of statistical significance was accepted at $p<0.05$.

\section{RESULTS}

All the cases in the study were re-evaluated by a reference observer. The grades, histological type, number, and percentage of the cases are presented in Table I. Two hundred and seven of the cases were Grade I, 59 were Grade II, and 4 were Grade III. One hundred and ninety-five $(72.2 \%)$ of the cases were female and 75 (27.8\%) were male.

Concordance among the observers (including five observers and a reference observer) was calculated with the Online Kappa Calculator. The free-marginal Kappa score was 0.88 (95\% confidence interval: 0.85-0.91).

The number of cases which were different with respect to grade as that evaluated by the reference observer was 41,40 , 47, 42, and 41, respectively (Table II). The Kappa concordance between the observers and the reference observer was very low. Kappa coefficient of Observer 2 had the lowest value. The Kappa scores of the other observers were between 0.40 and 0.60 . The mean numbers of mitotic figures which were found in 10 consecutive HPF by the observers for each case were $1.22,0.84,1.16,1.00$, and 1.24, respectively. In contrast, the mean number of mitoses noted by the reference observer was 1.94 (Table III). According to these results, Spearman's correlation coefficients between the observers and the reference observer in 10 consecutive HPF were $0.512,0.568$, $0.508,0.563$, and 0.563 , respectively. Scatter plot graphs between the observers and the reference observer, which show distribution of the number of mitoses in 10 consecutive HPF, are given in Figure 1. After the evaluation of mitotic activity

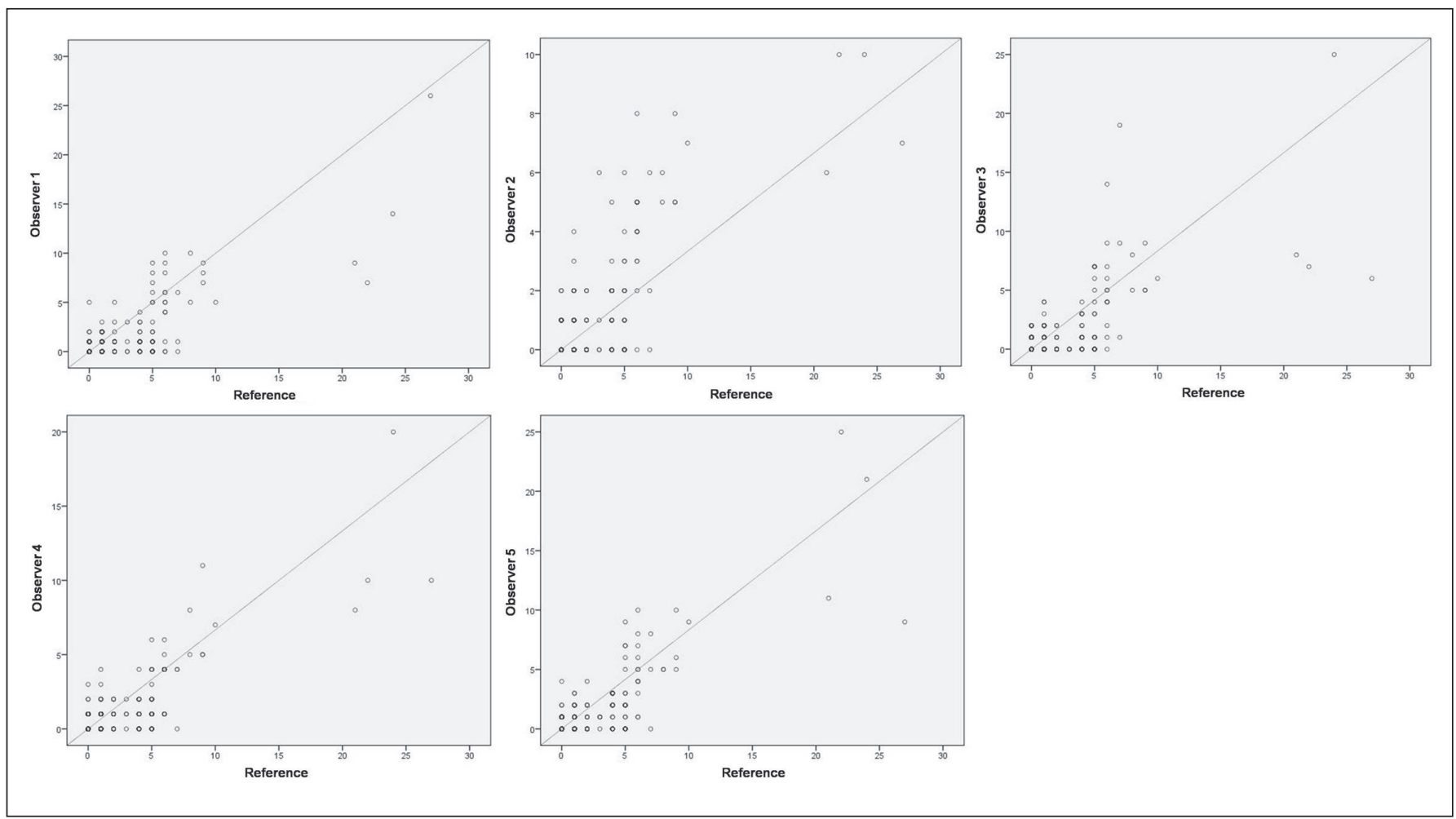

Figure 1: Scatter plot graphs between the observers and the reference observer, which show distribution of the number of mitoses in 10 consecutive HPF. 
Saygin I. et al: Mitosis and Grade Concordance

Table I: Histopathologic Characteristics of the Cases

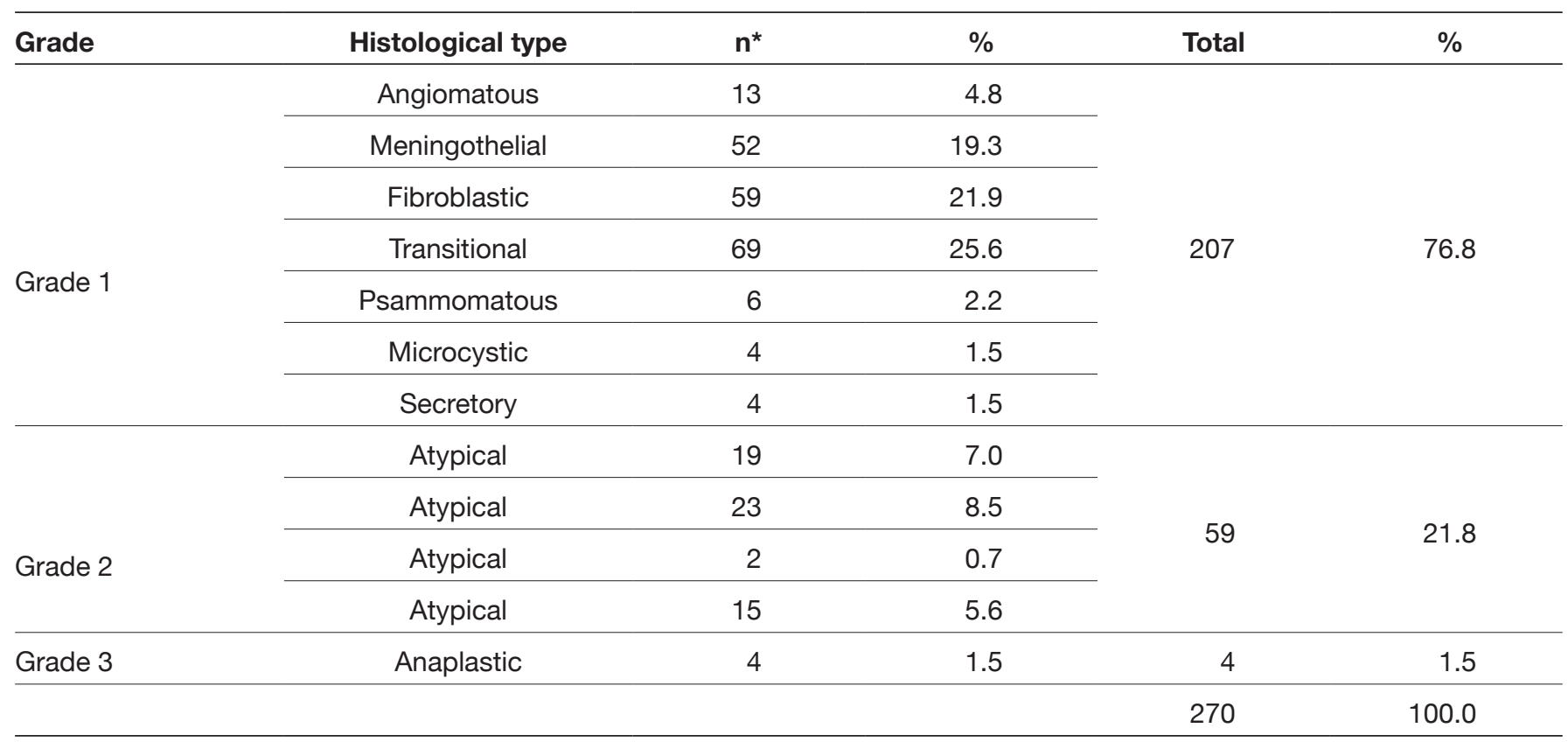

*The number of cases.

Table II: Comparison of the Histological Grades Between Observers' and the Reference Observer

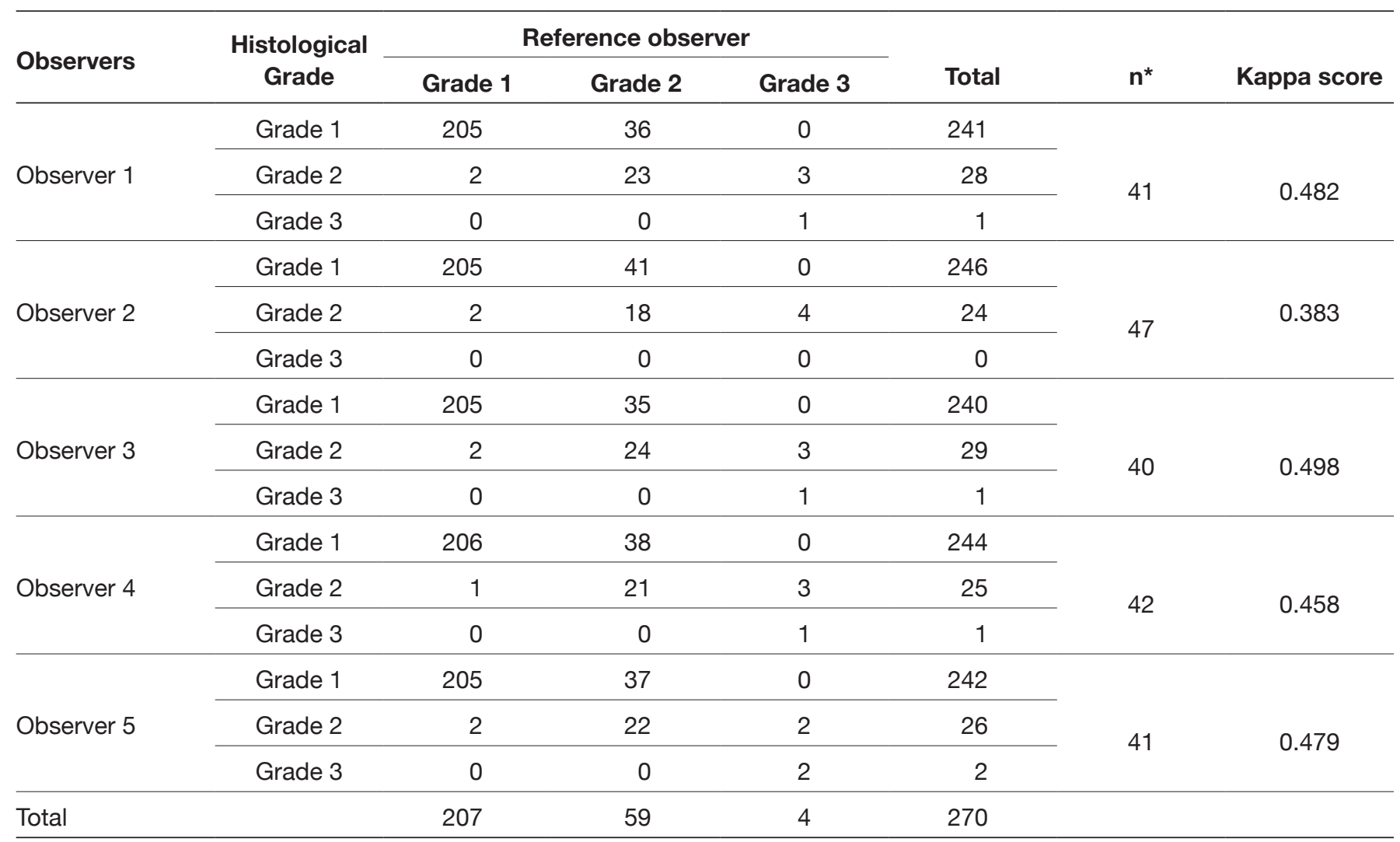

*The number of cases which were different with respect to grade. 
Saygin I. et al: Mitosis and Grade Concordance

Table III: Mean Mitotic Counts of the Observers And Reference Observer, and Spearman's Correlation Coefficients $p<0.001$

\begin{tabular}{|c|c|c|c|c|c|c|}
\hline & Observer 1 & Observer 2 & Observer 3 & Observer 4 & Observer 5 & $\begin{array}{c}\text { Reference } \\
\text { observer }\end{array}$ \\
\hline Minimum mitotic count & 0 & 0 & 0 & 0 & 0 & 0 \\
\hline Maximum mitotic count & 26 & 10 & 25 & 20 & 25 & 27 \\
\hline Mean mitotic count & $1.22 \pm 2.66$ & $0.84 \pm 1.78$ & $1.16 \pm 2.73$ & $1.00 \pm 2.09$ & $1.24 \pm 2.77$ & $1.94 \pm 3.45$ \\
\hline $\begin{array}{l}\text { Spearman's correlation } \\
\text { coefficient }\end{array}$ & 0.512 & 0.568 & 0.508 & 0.563 & 0.563 & \\
\hline
\end{tabular}

Table IV: Comparision of the Mean Numbers of Mitoses Between the Observers and the Reference Observer

\begin{tabular}{lccccc}
\hline & & \multicolumn{4}{c}{ Mean numbers of mitosis } \\
\cline { 3 - 5 } Observers & $\mathbf{n}^{*}$ & $\begin{array}{c}\text { Before full scan } \\
\text { method }\end{array}$ & $\begin{array}{c}\text { After full scan } \\
\text { method }\end{array}$ & Reference observer & $\mathbf{p}^{* *}$ \\
\hline Observer 1 & 41 & $1.71 \pm 2.79$ & $4.80 \pm 3.39$ & $5.83 \pm 4.84$ & $<0.001$ \\
\hline Observer 2 & 47 & $1.85 \pm 2.43$ & $3.96 \pm 2.27$ & $6.26 \pm 5.44$ & $<0.001$ \\
\hline Observer 3 & 40 & $1.55 \pm 1.98$ & $4.98 \pm 2.88$ & $5.88 \pm 5.20$ & $<0.001$ \\
\hline Observer 4 & 42 & $1.60 \pm 2.36$ & $4.48 \pm 2.47$ & $5.95 \pm 5.01$ & $<0.001$ \\
\hline Observer 5 & 41 & $1.95 \pm 2.23$ & $4.37 \pm 2.83$ & $5.41 \pm 4.45$ & $<0.001$ \\
\hline
\end{tabular}

*The number of cases with different grades between the observers and the reference observer.

${ }^{* *} p$ value for the comparison of the mean numbers of mitoses of the observers between the before and after the full scan method.

with the full scan method, the mean numbers of mitoses found by the observers in 10 consecutive HPF were 4.80, 3.96, 4.98, 4.48, and 4.37, respectively (Table IV).

Comparison of the results of the observers, before and after the full scan method, is demonstrated in Table V. In the first evaluation, 4-6 cases were defined as Grade II by the observers. Whereas, 23-27 cases were defined as Grade II after the full scan method. Before and after full scan method, the results showed statistically significant difference $(p<0.001)$.

Kappa scores of the results of the reference observer and the results found after full scan method by the observers are demonstrated in Table VI. According to these data, the kappa score was low and concordance was not very good, despite using full scan method. However, when the data were analyzed in a raw form, it was seen that most of the cases, defined as Grade I in the previous examination, were defined as Grade II after using full scan method. In addition, a few cases were found as of higher grade by some observers compared to the grades by the reference observer.

\section{DISCUSSION}

For various types of tumors, several studies have been done to determine the grade and treatment decision. In most of them, the main aim has been to determine the proliferation capacity of the tumor cells. Accurate determination of mitotic activity has been the focus of these studies. We investigated how to reduce the interobserver variability in meningioma grading.

In our study, when the data were analyzed in a raw form, it was expected that the interobserver difference in the tumor grade would be quite high. However, free-marginal kappa score was very high and grade concordance was very good among the observers (including five observers and reference observer). The grade concordance was evaluated with kappa analysis for each observer, and a moderate agreement was found between the observers and the reference observer, except Observer 2. The moderate agreement between the reference observer and the observers has suggested that there may be mitotic activities that cannot be detected by the observers.

The number of mitoses found by the observers was compared with those found by the reference observer. The mean mitotic counts of all the observers were lower than that of the reference observer. This result showed us again that observers cannot detect some mitotic activities. According to Spearman's correlation test, although the mean mitosis count in 10 consecutive HPF was the least, Observer 2 best correlated with the reference observer. Nevertheless, Observer 2 had the worst grade concordance with the reference observer. Therefore, there seems to be no direct relationship between the number of mitoses and grade. However, due to undetectable mitotic activity affecting the mitosis count in 10 consecutive HPF, it affects the grade indirectly. In that case, kappa analysis for grade concordance is more accurate than Spearman's correlation test. 
Saygin I. et al: Mitosis and Grade Concordance

Table V: Comparison of the Histological Grades of the Observers, Before and After the Full Scan Method

\begin{tabular}{|c|c|c|c|c|c|}
\hline \multirow[b]{2}{*}{ Observers } & \multirow{2}{*}{$\begin{array}{c}\text { Histological grade } \\
\begin{array}{c}\text { Before full scan } \\
\text { method }\end{array}\end{array}$} & \multicolumn{3}{|c|}{ After full scan method } & \multirow[b]{2}{*}{$\mathbf{p}$} \\
\hline & & Grade 1 & Grade 2 & Total (\%) & \\
\hline \multirow{3}{*}{ Observer 1} & Grade 1 & 17 & 19 & $36(87.8)$ & \multirow{3}{*}{$<0.001$} \\
\hline & Grade 2 & 0 & 5 & $5(12.2)$ & \\
\hline & Total (\%) & $17(41.5)$ & $24(58.5)$ & $41(100)$ & \\
\hline \multirow{3}{*}{ Observer 2} & Grade 1 & 22 & 19 & $41(87.2)$ & \multirow{3}{*}{$<0.001$} \\
\hline & Grade 2 & 0 & 6 & $6(12.8)$ & \\
\hline & Total (\%) & $22(46.8)$ & $25(53.2)$ & $47(100.0)$ & \\
\hline \multirow{3}{*}{ Observer 3} & Grade 1 & 15 & 20 & $35(87.5)$ & \multirow{3}{*}{$<0.001$} \\
\hline & Grade 2 & 0 & 5 & $5(12.5)$ & \\
\hline & Total (\%) & $15(37.5)$ & $25(62.5)$ & $40(100.0)$ & \\
\hline \multirow{3}{*}{ Observer 4} & Grade 1 & 14 & 24 & $38(90.5)$ & \multirow{3}{*}{$<0.001$} \\
\hline & Grade 2 & 1 & 3 & $4(9.5)$ & \\
\hline & Total (\%) & $15(35.7)$ & $27(64.3)$ & $42(100.0)$ & \\
\hline \multirow{3}{*}{ Observer 5} & Grade 1 & 17 & 20 & $37(90.2)$ & \multirow{3}{*}{$<0.001$} \\
\hline & Grade 2 & 1 & 3 & $4(9.8)$ & \\
\hline & Total (\%) & 18 (43.9) & $23(56.1)$ & $41(100.0)$ & \\
\hline
\end{tabular}

Table VI: Comparison of the Histological Grades Between the Reference Observer and the Observers After the Full Scan Method

\begin{tabular}{|c|c|c|c|c|c|c|c|}
\hline \multirow[b]{2}{*}{ Observers } & \multirow{2}{*}{$\begin{array}{c}\text { Histological grade } \\
\begin{array}{c}\text { After full scan } \\
\text { method }\end{array}\end{array}$} & \multicolumn{3}{|c|}{ Reference observer } & \multirow[b]{2}{*}{ Total (\%) } & \multirow{2}{*}{$\begin{array}{l}\text { Kappa } \\
\text { score }\end{array}$} & \multirow[b]{2}{*}{$\mathbf{p}^{*}$} \\
\hline & & Grade 1 & Grade 2 & Grade 3 & & & \\
\hline \multirow{3}{*}{ Observer 1} & Grade 1 & 0 & 17 & 0 & $17(41.5)$ & \multirow{3}{*}{-0.152} & \multirow{3}{*}{0.060} \\
\hline & Grade 2 & 2 & 19 & 3 & $24(58.5)$ & & \\
\hline & Total (\%) & $2(4.9)$ & $36(87.8)$ & $3(7.3)$ & $41(100.0)$ & & \\
\hline \multirow{3}{*}{ Observer 2} & Grade 1 & 0 & 22 & 0 & $22(46.8)$ & \multirow{3}{*}{-0.154} & \multirow{3}{*}{0.021} \\
\hline & Grade 2 & 2 & 19 & 4 & 25 (53.2) & & \\
\hline & Total (\%) & $2(4.3)$ & $41(87.2)$ & $4(8.5)$ & $47(100.0)$ & & \\
\hline \multirow{3}{*}{ Observer 3} & Grade 1 & 0 & 15 & 0 & $15(37.5)$ & \multirow{3}{*}{-0.151} & \multirow{3}{*}{0.083} \\
\hline & Grade 2 & 2 & 20 & 3 & $25(62.5)$ & & \\
\hline & Total (\%) & $2(5.0)$ & $35(87.5)$ & $3(7.5)$ & $40(100.0)$ & & \\
\hline \multirow{3}{*}{ Observer 4} & Grade 1 & 1 & 14 & 0 & $15(35.7)$ & \multirow{3}{*}{0.012} & \multirow{3}{*}{0.860} \\
\hline & Grade 2 & 0 & 24 & 3 & 27 (64.3) & & \\
\hline & Total (\%) & $1(2.4)$ & $38(90.5)$ & $3(7.1)$ & $42(100.0)$ & & \\
\hline \multirow{3}{*}{ Observer 5} & Grade 1 & 1 & 17 & 0 & $18(43.9)$ & \multirow{3}{*}{-0.033} & \multirow{3}{*}{0.672} \\
\hline & Grade 2 & 1 & 20 & 2 & $23(56.1)$ & & \\
\hline & Total (\%) & $2(4.9)$ & 37 (90.2) & $2(4.9)$ & $41(100.0)$ & & \\
\hline
\end{tabular}

${ }^{*} p$ value indicates no significant difference between the histological grades. 
In the second phase of the study, the observers re-examined the cases they found as different grade from the reference observer, by the full scan method. After this method, the observers changed grade in more than $50 \%$ of the cases. In other words, they changed most of the Grade I cases to Grade II. Similar to our study, Moradi et al. showed that the grade of some cases changed after re-examination of the mitotic figures (10). In our study, there was a statistically significant difference in the number of mitoses and grade, before and after the full scan method. It also showed that the full scan method was more useful.

Although there was a statistically significant increase in the mitotic count concordance by the full scan method, this situation was not reflected in grade concordance. Kappa score of grade concordance and $p$ values between the observers and the reference observer were found meaningless by the full scan method. According to these findings, the first reason of the grade discordance is that the number of mitotic figures found by the observers was less than the reference observer. The second and more important reason is that the number of mitoses found by the observers in 10 consecutive HPF was less than 4 for Grade II and 20 for Grade III. In the light of these findings, we can say that the observers begun to detect mitotic activity more successfully, but they had difficulties in fitting 4 or more mitotic activities into 10 consecutive HPF.

All the observers described the tumors as different grade than the reference observer in more than 40 cases (more than 15\% of all cases). The total number of these cases was 52 . When the anaplastic meningioma and the cases defined as higher grade than the reference observer were excluded (10 cases totally), the observers described the remaining 42 cases as Grade I instead of Grade II. In these 42 cases, the arithmetic mean of the mitotic figures, detected by the reference observer in the entire slides, was approximately 16 . As a result, when the number of mitotic figures in the entire slide was less than 16 , observers had difficulties in finding the mitotic figures. Therefore, the observers found lower mitotic activity in 10 consecutive HPF, and they defined most of the cases as lower grade.

In the study by Commins et al., the reliability of the mitosis counting method was controversial, due to some factors such as tissue fixation, section thickness problems, the differences in observer experience, variability of the mitotic count from field to field, and differences in microscopes (3).

Mitotic figures are generally counted in the most hypercellular area of the tumor, and there is no rule about how this area is chosen by the pathologist. In some studies, it is noticeable that mitosis was counted in areas with high mitotic activity (1). In our study, using the same type of microscope and examining the entire slide with full scan method were very effective in determining the accurate number of mitoses. In addition to what Commins et al. said, the possible reasons of differences in mitosis count in daily routine practice may be as follows: not determining the area with high mitotic activity, not examining carefully the entire slide or the slides which include low mitotic activity, confusion of mitotic figures with apoptotic cells or pyknotic nuclei, and insufficient time allowed for accurate counting.

\section{CONCLUSION}

The concordance of the mean number of mitoses between the observers and the reference observer was poor. However, we conclude that the interobserver variability can be reduced by full scan method. Since only one mitotic activity may change the grade, we recommend that the mitosis count should be performed at $400 \times$ objective on $\mathrm{H}$ and $\mathrm{E}$ slides. Especially if we notice mitosis activity, but cannot detect 4 or more mitosis in 10 consecutive HPF, we should be more careful. According to our study, if there are less than 16 mitotic figures throughout the slide, these tumors are defined as Grade I, instead of Grade II. In other words, in the cases which include low number of mitotic figures, it is more difficult to find the 10 consecutive HPF including 4 or more mitosis. Therefore, these cases are easily defined as Grade I.

In conclusion, examining the $\mathrm{H}$ and $\mathrm{E}$ stained slides by the full scan method helps us determine the true histologic grade of meningiomas in most cases, who would receive an additional treatment.

\section{REFERENCES}

1. Backer-Grøndahl T, Moen BH, Torp SH. The histopathological spectrum of human meningiomas. Int $\mathrm{J}$ Clin Exp Pathol 5(3):231-242, 2012

2. Colman H, Giannini C, Huang L, Gonzalez J, Hess K, Bruner J, Fuller G, Langford L, Pelloski C, Aaron J et al. "Assessment and prognostic significance of mitotic index using the mitosis marker phospho-histone h3 in low and intermediate-grade infiltrating astrocytomas," Am J Surg Pathol 30(5):657-664, 2006

3. Commins DL, Atkinson RD, Burnett ME: Review of meningioma histopathology. Neurosurg Focus 23(4):3-9, 2007

4. Duregon E, Cassenti A, Pittaro A, Ventura L, Senetta R, Ruda R, Cassoni P: Better see to better agree: Phosphohistone $\mathrm{H} 3$ increases interobserver agreement in mitotic count for meningioma grading and imposes new specific thresholds. Neuro Oncol 17(5):663-669, 2015

5. Gallagher MJ, Jenkinson MD, Brodbelt AR, Mills SJ, Chavredakis E: WHO grade 1 meningioma recurrence: Are location and Simpson grade still relevant? Clin Neurol Neurosurg 141:117-121, 2016

6. Gousias K, Schramm J, Simon M: The Simpson grading revisited: Aggressive surgery and its place in modern meningioma management. J Neurosurg 125:551-560, 2016

7. Ho DM, Hsu CY, Ting LT, Chiang H: Histopathology and MIB-1 labeling index predicted recurrence of meningiomas: A proposal of diagnostic criteria for patients with atypical meningioma. Cancer 94:1538-1547, 2002

8. Kim YJ, Ketter R, Steudel WI, Feiden W: Prognostic significance of the mitotic index using the mitosis marker anti phosphohistone $\mathrm{H} 3$ in meningiomas. Am J Clin Pathol 128:118-125, 2007

9. Louis DN, Perry A, Reifenberger G, von Deimling A, FigarellaBranger D, Cavenee WK, Ohgaki H, Wiestler OD, Kleihues P, Ellison DW: The 2016 World Health Organization Classification of tumors of the central nervous system: A summary. Acta Neuropathol 131:803-820, 2016 
10. Moradi A, Semnani V, Djam H, Tajodini A, Zali AR, Ghaemi K, Nikzad N, Madani-Civi M: Pathodiagnostic parameters for meningioma grading. J Clin Neurosci 15(12):1370-1375, 2008

11. Ostrom QT, Gittleman H, Fulop J, Liu M, Blanda R, Kromer C, Wolinsky Y, Kruchko C, Barnholtz-Sloan JS: CBTRUS statistical report: Primary brain and central nervous system tumors diagnosed in the United States in 2008-2012. Neuro Oncol 17 Suppl 4:iv1-iv62, 2015

12. Perry A, Stafford SL, Scheithauer BW, Suman VJ, Lohse CM: Meningioma grading: An analysis of histologic parameters. Am J Surg Pathol 21(12):1455-1465, 1997

13. Randolph JJ: Online Kappa Calculator (Computer software). Retrieved from http://justus.randolph.name/kappa.

14. Ribalta T, McCutcheon IE, Aldape KD, Bruner JM, Fuller GN: The mitosis-specific antibody anti-phosphohistone-h3 facilitates rapid reliable grading of meningiomas according to who 2000 criteria. Am J Clin Pathol 28(11):1532-1536, 2004
15. Rogers L, Gilbert M, Vogelbaum MA: Intracranial meningiomas of atypical (WHO gradell) histology. J Neurooncol 99:393-405, 2010

16. Talacchi A, Muggiolu F, De Carlo A, Nicolato A, Locatelli F, Meglio M: Recurrent atypical meningiomas: Combining surgery and radiosurgery in one effective multimodal treatment. World Neurosurg 87:565-572, 2016

17. Tellez D, Balkenhol M, Otte-Holler I, van de Loo R, Vogels R, Bult P, Wauters C, Vreuls W, Mol S, Karssemeijer N, Litjens G, van der Laak J, Ciompi F: Whole-slide mitosis detection in H\&E breast histology using $\mathrm{PHH} 3$ as a reference to train distilled stain invariant convolutional networks. IEEE Trans Med Imaging 37(9):2126-2136, 2018

18. Van Alkemade H, De Leau M, Dieleman EM, Kardaun JW, Van Os R, Vandertop WP, Van Furth WR, Stalpers LJ: Impaired survival and long-term neurological problems in benign meningioma. Neuro Oncol 14:658-666, 2012 